\title{
A PROJECTIVE GENERALIZATION OF METRICALLY DEFINED ASSOCIATE SURFACES*
}

\author{
BY \\ M. L. MACQUEEN

\section{INTRODUCTION}

In the metric differential geometry of surfaces in ordinary space, two surfaces are said by Bianchi to be associate $\dagger$ if the tangent planes at corresponding points are parallel and if the asymptotic curves on either surface correspond to a conjugate net on the other.

It is the purpose of this paper to develop a projective generalization of the relation of associateness of surfaces. Since associate surfaces are parallel in the metric sense, it will first be necessary to provide a projectively defined substitute for the property of metric parallelism. We shall employ as the basis of our study in this paper a projective generalization of euclidean parallelism of surfaces which the author has developed in his Chicago doctoral dissertation.

In $\$ 2$, after stating a definition of projective parallelism of surfaces and briefly explaining this idea, we introduce a canonical form of our system of differential equations employed in the study of projectively parallel surfaces in ordinary space. In $\$ 3$ we formulate a definition of projectively associate surfaces and investigate to some extent their properties and relations. A more general type of associateness which may be conveniently termed modified projective associateness is introduced in $\S 4$, and a somewhat different canonical form of our system of differential equations is employed in its study. Finally, in $\$ 5$, we consider a rather general completely integrable system of partial differential equations, namely, the system for two surfaces in the general analytic one-to-one point correspondence in ordinary projective space $S_{3}$, and a group of transformations that leaves this configuration invariant. We then reduce this system of equations to a new canonical form, and employ it to continue briefly the study of modified projective associateness introduced in the preceding section.

\footnotetext{
* Presented to the Society, September 7, 1934; received by the editors April 8, 1934.

$\dagger$ Eisenhart, Differential Geometry, Ginn and Company, 1909, p. 378. Hereinafter cited as Eisenhart. See also Bianchi, Lezioni di Geometria Differenziale (3d edition), vol. 2, p. 10.
} 


\section{Projective Parallelism of SURfaces}

In formulating a projective generalization of metric parallelism of surfaces, ${ }^{*}$ we begin by replacing the metric normal congruence by the projective normal congruence, and so consider two surfaces $S_{x}, S_{y}$, in ordinary projective space $S_{3}$, with a common projective normal congruence. The developables of this congruence intersect both surfaces in the projective lines of curvature, which form conjugate nets. We then demand, in analogy to the metric parallelism of the tangent planes, that the tangent planes at corresponding points of the two surfaces intersect on a fixed plane. Two surfaces so related are said to be parallel in the projective sense.

For the basis of our study of projective parallelism we employ one of the well known transformations of surfaces, namely, the fundamental transformation. Two surfaces are said to be in the relation of a fundamental transformation, or transformation $F \dagger$, in case their points are in a one-to-one correspondence such that the lines joining corresponding points form a congruence whose developables intersect both surfaces in conjugate nets, neither surface being a focal surface of the congruence. The congruence is called the conjugate congruence of the transformation because it is conjugate to both nets. The tangent planes at corresponding points of the two surfaces intersect in the lines of the harmonic congruence of the transformation, which is harmonic to both nets. By choosing the projective normal congruence as the common conjugate congruence of the transformation $F$, we provide, as previously stated, a projective substitute for the metric normal congruence. Furthermore, we assume that the developables of the harmonic congruence are indeterminate, that is, that corresponding tangent planes of the two surfaces intersect in the lines of a fixed plane. This assumption affords us a projective substitute for the metric parallelism of the tangent planes. Our definition of projective parallelism may now be stated in the following way:

Two surfaces $S_{x}, S_{y}$ are said to be projectively parallel in case they are in the relation of a fundamental transformation with the projective normal congruence as the conjugate congruence and with the developables of the harmonic congruence indeterminate.

In order to represent analytically the definition which we have introduced, let us consider two projectively parallel surfaces $S_{x}, S_{y}$ with the respective. parametric vector equations

$$
x=x(u, v), y=y(u, v) .
$$

*M.L. MacQueen, A Projective Generalization of Euclidean Parallelism of Surfaces, University of Chicago, December, 1933; unpublished doctoral dissertation. Hereinafter cited as Thesis.

† L. P. Eisenhart, Transformations of Surfaces, Princeton University Press, 1923, p. 34 et seq. 
The four coordinates $x$ and the four coordinates $y$ form four pairs of solutions of a completely integrable system of differential equations of the form

$$
\begin{aligned}
x_{u u} & =p x+\alpha x_{u}+\beta x_{v}+L y, \\
x_{u v} & =c x+a x_{u}+b x_{v}, \\
x_{v v} & =q x+\gamma x_{u}+\delta x_{v}+N y, \\
y_{u} & =f x+m x_{u}+A y, \\
y_{v} & =g x+n x_{v}+B y
\end{aligned}
$$

$(m n L N \neq 0)$,

where the notation here employed is similar to that used by Lane in his recent book.*

Before stating the conditions which characterize this system we remark that in order to treat $S_{x}, S_{y}$ in a symmetrical manner we see that $x, y$ satisfy a system of equations of the form (1), but with the roles of $x$ and $y$ interchanged. The coefficients of such a system will be indicated by dashes and will be given later. In order that $S_{y}$ may be non-developable we shall suppose that $\bar{L} \vec{N} \neq 0$.

System (1) is characterized analytically by the following conditions:

(a) $\alpha+b+A+(\log N)_{u}-3(\log r)_{u} / 2-2(\log R)_{u}=0$,

(b) $r / r+\alpha+(\log r)_{u} / 2=0$,

(2)

(c) $\bar{r}=n r / m$,

(d) $f / m=-\left[\log (m n)^{1 / 2} R / L\right]_{u}$,

(e) $m(1-n) \mathfrak{B}^{\prime 2}+n r(1-m) \mathfrak{E}^{\prime 2}+m_{v}\left(\mathfrak{B}^{\prime}+m_{v} /(4 m)\right)$

$$
+n_{u} r\left(\mathbb{S}^{\prime}+n_{u} /(4 n)\right)=0
$$

and by the counterpart of (a), (b), and (d) in the substitution

$$
\left(\begin{array}{llllllllllllll}
u & a & c & f & m & p & s & \alpha & \beta & A & L & M & r & R \\
v & b & c & g & n & q & t & \delta & \gamma & B & N & M & 1 / r & r R
\end{array}\right) .
$$

The invariants $\mathfrak{B}^{\prime}, \mathfrak{S}^{\prime}, R$ of Green, and the invariant $r$ of Eisenhart, appearing in equations (2), are expressed for the projective lines of curvature on $S_{x}$ in terms of the coefficients of system (1) by the following formulas:

$$
\begin{aligned}
8 \mathfrak{B}^{\prime} & =4 a+2 N \beta / L-2 \delta+(\log N / L)_{v}, \\
8 \mathfrak{S}^{\prime} & =4 b+2 L \gamma / N-2 \alpha+(\log L / N)_{u}, \\
R & =L \mathscr{B}^{\prime 2} / N+\mathfrak{C}^{\prime 2}, \\
r & =N / L .
\end{aligned}
$$

* Lane, Projective Differential Geometry of Curves and Surfaces, University of Chicago Press, 1932, p. 183. Hereinafter cited as Lane. 
Conditions (2) (a) imply that the line $x_{u} x_{v}$ is the reciprocal of the projective normal of $S_{x}$ at $P_{x}$, and conditions (b) and (a) imply that the line $x y$ is the projective normal of $S_{x}$ at $P_{x}$; condition (c) implies that the tangent planes at corresponding points of $S_{x}, S_{y}$ intersect in the lines of a fixed plane; conditions (c) and (d) imply that the line $y_{u} y_{v}$ is the reciprocal of the projective normal of $S_{v}$ at $P_{y}$, and conditions (c), (d), and (e) imply that the line $x y$ is the projective normal of $S_{y}$ at $P_{y}$.

It may be remarked that the choice of the proportionality factors which leads to our canonical form is precisely that which gives Fubini's normal coordinates.

The integrability conditions for system (1) are given by the following equations and those obtainable therefrom by the substitution (3):

$$
\begin{aligned}
& a_{u}+a b+c=\alpha_{v}+\beta \gamma, \\
& b_{u}+b^{2}+a \beta=\beta_{v}+b \alpha+\beta \delta+p+n L, \\
& c_{u}+b c+a p=p_{v}+c \alpha+q \beta+g L, \\
& g_{u}+c n+f B=f_{v}+c m+g A, \\
& a n+m B+g=m_{v}+a m, \\
& a L=L_{v}+B L+\beta N, \quad A_{v}=B_{u} .
\end{aligned}
$$

The coefficients of the equations corresponding to (1) when the roles of $x$ and $y$ are interchanged are given by

$$
\begin{aligned}
\bar{p} & =A_{u}+m L-A\left(m_{u} / m+f / m+\alpha\right)-m \beta B / n, \\
\bar{\alpha} & =\alpha+f / m+m_{u} / m+A, \quad \bar{\beta}=m \beta / n, \\
\bar{L} & =-m\left(\alpha f / m+\beta g / n+(f / m)^{2}-p-(f / m)_{u}\right), \\
\bar{c} & =A_{v}-A\left(m_{v} / m+a\right)-B(f / n+m b / n), \\
& =B_{u}-B\left(n_{u} / n+b\right)-A(g / m+n a / m), \\
\bar{a} & =a+m_{v} / m=B+g / m+n a / m, \\
\bar{b} & =b+n_{u} / n=A+f / n+m b / n, \\
\bar{q} & =B_{v}+n N-B\left(n_{v} / n+g / n+\delta\right)-n \gamma A / m, \\
\bar{\gamma} & =n \gamma / m, \quad \bar{\delta}=\delta+g / n+n_{v} / n+B, \\
\bar{N} & =-n\left(\delta g / n+\gamma f / m+(g / n)^{2}-q-(g / n)_{v}\right), \\
\bar{f} & =-A / m, \quad \bar{m}=1 / m, \quad \bar{A}=-f / m, \\
\bar{g} & =-B / n, \quad \bar{n}=1 / n, \quad \bar{B}=-g / n .
\end{aligned}
$$

The developables of the projective normal congruence intersect $S_{x}$ and $S_{y}$ in parametric conjugate nets which are the projective lines of curvature thereon, the foci $P_{\eta}, P_{5}$ of a projective normal being given by 


$$
\eta=y-m x, \quad \zeta=y-n x .
$$

The differential equation of the asymptotic curves on $S_{x}$ is

$$
L d u^{2}+N d v^{2}=0,
$$

and the asymptotic curves on $S_{y}$ are given by the equation

$$
\bar{L} d u^{2}+\bar{N} d v^{2}=0 \text {. }
$$

\section{Projectively associate surfaces}

The projective generalization of metric parallelism of surfaces summarized in the preceding section will now be employed in formulating a definition of projectively associate surfaces. In analogy to the metric definition of associate surfaces, two surfaces $S_{x}, S_{y}$, in ordinary projective space, will be called projectively associate if they are projectively parallel and if the asymptotic curves on either surface correspond to a conjugate net on the other.

A necessary and sufficient condition that the asymptotic curves on one of two projectively parallel surfaces $S_{x}, S_{y}$ correspond to a conjugate net on the other is

$$
L \bar{N}+\bar{L} N=0,
$$

i.e., the harmonic invariant of the asymptotic curves on the two surfaces vanishes. With the aid of (2) (c) this condition is seen to be equivalent to

$$
m=-n \text {. }
$$

By means of (7), condition (11) shows that $P_{y}$ is the harmonic conjugate of $P_{x}$ with respect to the two focal points of a projective normal. Thus we reach the following conclusion:

If two surfaces $S_{x}, S_{y}$ are projectively parallel, a necessary and sufficient condition that they be projectively associate is that corresponding points on a projective normal separate harmonically the foci thereon.

The Laplace-Darboux point invariants, $H, K$, the Weingarten invariants $W^{(u)}, W^{(v)}$, and the tangential invariants $\mathfrak{E}, \Omega$ are given for the projective lines of curvature on $S_{x}$ in terms of the coefficients of system (1) by the formulas

$$
\begin{aligned}
H & =c+a b-a_{u}, \quad K=c+a b-b_{v}, \\
W^{(u)} & =2 b_{v}+a_{u}-\delta_{u}-B_{u}-(\log L)_{u v}, \\
W^{(v)} & =2 a_{u}+b_{v}-\alpha_{v}-A_{v}-(\log N)_{u v}, \\
\mathfrak{E} & =K+W^{(u)}=a_{u}+\beta \gamma-B_{u}-(\log L)_{u v}, \\
& =N\left(\beta_{u}+\beta b-\beta A-\beta(\log L)_{u}\right) / L, \\
\Omega & =H+W^{(v)}=b_{v}+\beta \gamma-A_{v}-(\log N)_{u v}, \\
& =L\left(\gamma_{v}+\gamma a-\gamma B-\gamma(\log N)_{v}\right) / N .
\end{aligned}
$$


The corresponding invariants, indicated by dashes, for the projective lines of curvature on $S_{y}$, projectively parallel to $S_{x}$, are found* to have the following expressions:

$$
\begin{aligned}
\bar{H} & =H-\left(\log m^{3} n\right)_{u v} / 2, & \bar{K} & =K-\left(\log m n^{3}\right)_{u v} / 2, \\
\bar{W}^{(u)} & =W^{(u)}+\left(\log m n^{3}\right)_{u v} / 2, & \overline{W^{(v)}} & =W^{(v)}+\left(\log m^{3} n\right)_{u v} / 2 \\
\overline{\mathfrak{S}} & =\mathfrak{W}, & \bar{\Omega} & =\Re .
\end{aligned}
$$

It is evident from (2) (d) that

$$
(f / m)_{v}=(g / n)_{u} .
$$

By using (14) and the integrability conditions (5) a simple calculation is made which shows that in case $m=-n$ it follows that $a_{u}=b_{v}$, and the projective lines of curvature on $S_{x}$ have equal point invariants. Moreover, in this case equations (13) show that the projective lines of curvature on $S_{y}$ also have equal point invariants. We therefore reach the following conclusion:

If two surfaces $S_{x}, S_{y}$ are projectively associate, the projective lines of curvature on each surface have equal point invariants.

We shall now investigate the conjugate nets on each of two projectively associate surfaces to which correspond the asymptotic curves on the other. When use is made of (10), equation (8), which defines the asymptotic curves on $S_{x}$, may be written

$$
\bar{L} d u^{2}-\bar{N} d v^{2}=0 .
$$

This is the differential equation of the associate conjugate net of the projective lines of curvature on $S_{y}$, that is, the conjugate net whose tangents at each point of the surface $S_{\nu}$ separate harmonically the tangents to the projective lines of curvature.

Similarly, by use of (10), we may write equation (9) in the form

$$
L d u^{2}-N d v^{2}=0,
$$

which shows that the asymptotic curves on $S_{y}$ correspond to the associate conjugate net of the projective lines of curvature on $S_{x}$. We may therefore state the following theorem:

If two surfaces $S_{x}, S_{y}$ are projectively associate, and if the parametric net on each is the projective lines of curvature, then the asymptotic curves on either surface correspond to the associate conjugate net of the parametric conjugate net on the other.

* Thesis. 
An interesting property of a conjugate net is isothermal conjugacy, the condition for which is $W^{(u)}=W^{(v)}$ or $(\log r)_{u v}=0$. Let the projective lines of curvature on $S_{x}$ be an isothermally conjugate net, and let $S_{y}$ be projectively associate to $S_{x}$. From (10) or (12) it is then easy to obtain the following result:

If the projective lines of curvature are isothermally conjugate on one of two projectively associate surfaces, they are also isothermally conjugate on the other.

In this case the projective lines of curvature on $S_{x}$ and $S_{y}$ are called $J$ nets, since they are isothermally conjugate and have equal point invariants.

\section{MODIFIED PROJECTIVE ASSOCIATENESS OF SURFACES}

In this section we shall drop the assumption that the common conjugate congruence of the transformation $F$ is the projective normal congruence, and shall employ in its place a general conjugate congruence. The configuration composed of two surfaces in ordinary space in the relation of a fundamental transformation having a general conjugate congruence and with the developables of the harmonic congruence indeterminate leads us to a characterization of surfaces which are projectively parallel in a modified* sense. We shall use this type of parallelism in formulating our definition of modified projectively associate surfaces.

For the analytic basis of our work a somewhat different canonical form of the basic system of differential equations is employed. If $S_{x}, S_{y}$ are a pair of surfaces projectively parallel in the modified sense, then the four coordinates $x$ and the four coordinates $y$ form four pairs of solutions of a completely integrable system of differential equations* of the form

$$
\begin{aligned}
x_{u u} & =L(x+y)+\alpha x_{u}+\beta x_{v}, \\
x_{u v} & =a x_{u}+b x_{v}, \\
x_{v v} & =N(x+y)+\gamma x_{u}+\delta x_{v}, \\
y_{u} & =m x_{u}, \quad y_{v}=n x_{v}
\end{aligned}
$$

The integrability conditions for this system are found to be

$$
\begin{aligned}
& a_{u}+a b=\alpha_{v}+\beta \gamma, \quad b_{v}+a b=\delta_{u}+\beta \gamma, \\
& b_{u}+b^{2}+a \beta=\beta_{v}+b \alpha+n L+\beta \delta+L, \\
& a_{v}+a^{2}+b \gamma=\gamma_{u}+a \delta+m N+\alpha \gamma+N, \\
& L_{v}=a L-\beta N, \quad N_{u}=b N-\gamma L, \\
& m_{v}=a(n-m), \quad n_{u}=b(m-n) .
\end{aligned}
$$

* Thesis. 
The coefficients of the equations corresponding to (16), when the roles of $x$ and $y$ are interchanged, are indicated by dashes and are given by the following expressions:

$$
\begin{aligned}
& \bar{L}=m L, \quad \bar{\alpha}=\alpha+m_{u} / m, \quad \bar{\beta}=m \beta / n \\
& \bar{a}=n a / m, \quad \bar{b}=m b / n, \quad \bar{m}=1 / m, \quad \bar{n}=1 / n, \\
& \bar{N}=n N, \quad \bar{\gamma}=n \gamma / m, \quad \bar{\delta}=\delta+n_{v} / n .
\end{aligned}
$$

We shall assume that $\bar{L} \bar{N} \neq 0$ in order that $S_{y}$ may be non-developable.

The focal points $P_{y}, P_{\zeta}$ of a line $x y$ joining corresponding points $P_{x}, P_{y}$ of two surfaces $S_{x}, S_{y}$ are defined by

$$
\eta=y-m x, \quad \zeta=y-n x .
$$

Several results similar to those in the preceding section will now be given. Inasmuch as the proofs of these results run parallel to those in $\$ 3$ they will be omitted in this section. Precisely as in the preceding section, a necessary and sufficient condition that the asymptotic curves on either of two modified projectively parallel surfaces $S_{x}, S_{\nu}$ correspond to a conjugate net on the other is found to be given by the condition $m=-n$. Hence the following result is readily obtained.

If two surfaces $S_{x}, S_{y}$ are projectively parallel in the modified sense, a necessary and sufficient condition that they be projectively associate in the same sense is that corresponding points $P_{x}, P_{y}$ of each line $x y$ separate harmonically the foci thereon.

Some of the invariants of the parametric conjugate net $N_{x}$ are found to have in our notation the following formulas:

$$
\begin{aligned}
H & =a b-a_{u}, \quad K=a b-b_{v}, \\
W^{(u)} & =2 b_{v}+a_{u}-\delta_{u}-(\log L)_{u v}, \\
W^{(v)} & =2 a_{u}+b_{v}-\alpha_{v}-(\log N)_{u v}, \\
\mathfrak{Q} & =K+W^{(u)}=a_{u}+\beta \gamma-(\log L)_{u v} \\
& =N\left(\beta_{u}+b \beta-\beta(\log L)_{u}\right) / L, \\
\Omega & =H+W^{(v)}=b_{v}+\beta \gamma-(\log N)_{u v} \\
& =L\left(\gamma_{v}+a \gamma-\gamma(\log N)_{v}\right) / N, \\
8 \mathscr{B}^{\prime} & =6 a-2 \delta-3(\log L)_{v}+(\log N)_{v}, \\
8 \mathfrak{C}^{\prime} & =6 b-2 \alpha-3(\log N)_{u}+(\log L)_{u} .
\end{aligned}
$$

By use of (17) and (18), the corresponding invariants for $N_{y}$, indicated by dashes, are given by the following expressions: 


$$
\begin{aligned}
\bar{H} & =H-(\log m)_{u v}, \\
\overline{W^{(u)}} & =W^{(u)}+(\log n)_{u v}, \\
\overline{\mathfrak{S}} & =\mathfrak{S}, \\
8 \mathfrak{\mathscr { B }}^{\prime} & =8 \mathfrak{B}^{\prime}+\left(\log m^{3} / n\right)_{v},
\end{aligned}
$$

$$
\begin{aligned}
\bar{K} & =K-(\log n)_{u v}, \\
\bar{W}^{(v)} & =W^{(v)}+(\log m)_{u v}, \\
\bar{\Omega} & =\Re, \\
8 \bar{\complement}^{\prime} & =8 \widetilde{C}^{\prime}+\left(\log n^{3} / m\right)_{u} .
\end{aligned}
$$

The following result can be easily established.

If two surface's $S_{x}, S_{y}$ are projectively associate in the modified sense, the parametric conjugate net on each surface has equal point invariants.

The asymptotic curves on $S_{x}$ and $S_{\nu}$ are determined by the same equations as in the preceding section. Precisely as in $\$ 3$ we arrive at the following result:

If two surfaces $S_{x}, S_{y}$ are projectively associate in the modified sense, and if the parametric net on each surface is conjugate, then the asymptotic curves on either surface correspond to the associate conjugate net of the parametric net on the other.

The Laplace transformed points, or ray points, of the point $P_{x}$ with respect to $N_{x}$ are given by

$$
x_{1}=x_{v}-a x, \quad x_{-1}=x_{u}-b x,
$$

and the ray points of $P_{y}$ are defined by

$$
y_{1}=n\left(x_{1}-a \eta / m\right), \quad y_{-1}=m\left(x_{-1}-b \zeta / n\right) .
$$

The ray points of the points $P_{\eta}, P_{5}$, defined by (19), are found to be

$$
\begin{aligned}
m_{u} \eta_{1} & =(n-m)\left(H \eta+m_{u} x_{1}\right), & (n-m) \eta_{-1} & =m_{u} \zeta, \\
(m-n) \zeta_{1} & =n_{v} \eta, & n_{v} \zeta_{-1} & =(m-n)\left(K \zeta+n_{v} x_{-1}\right),
\end{aligned}
$$

where $H, K$ are the point invariants of the net $N_{x}$. The points $x_{1}, y_{1}, \eta, \eta_{1}$ are collinear, as are also the points $x_{-1}, y_{-1}, \zeta, \zeta_{-1}$. The cross ratio* of the four points $x_{-1}, y_{-1}, \zeta, \zeta_{-1}$ is $-b n_{v} /(n K)$, and that of the points $x_{1}, y_{1}, \eta, \eta_{1}$ is $-a m_{u} /(m H)$. Hence we have the following theorem:

If two surfaces $S_{x}, S_{y}$ are projectively associate in the modified sense, the cross ratio of the four points $x_{1}, y_{1}, \eta, \eta_{1}$ is equal to that of the points $x_{-1}, y_{-1}, \zeta$, $\zeta_{-1}$, and the common value may be written $2 a b / H$.

\section{A CANONICAL FORM WITH PARTICULAR PARAMETRIC CURVES}

In this section we place as fundamental the well known system of differential equations used in the study of the configuration composed of two surfaces $S_{x}, S_{y}$ in ordinary projective space $S_{3}$, with their points in a one-to-one

\footnotetext{
* Lane, op. cit., p. 214, exercise 11.
} 
correspondence. We then reduce this system of equations to a canonical form so that every pair of integral surfaces is projectively associate in the modified sense, the surface $S_{x}$ being referred to its asymptotic net as parametric, and the curves on $S_{y}$, corresponding to the asymptotic curves on $S_{x}$, forming the parametric conjugate net.

Let

$$
x=x(u, v), \quad y=y(u, v)
$$

be the parametric vector equations of two surfaces $S_{x}, S_{y}$ in ordinary projective space. If these surfaces have their points in a one-to-one correspondence, such that corresponding points $P_{x}, P_{y}$ have the same curvilinear coordinates $u, v$, and such that each point $P_{y}$ does not lie in the tangent plane of $S_{x}$ at the corresponding point $P_{x}$, then $S_{x}, S_{y}$ are a pair of integral surfaces of a system of differential equations* of the form

$$
\begin{aligned}
x_{u u} & =p x+\alpha x_{u}+\beta x_{v}+L y, \\
x_{u v} & =c x+a x_{u}+b x_{v}+M y, \\
x_{v v} & =q x+\gamma x_{u}+\delta x_{v}+N y, \\
y_{u} & =f x+m x_{u}+s x_{v}+A y, \\
y_{v} & =g x+t x_{u}+n x_{v}+B y .
\end{aligned}
$$

The integrability conditions for this system are given by the following equations:

$$
\begin{aligned}
a_{u}+a b+c+m M & =\alpha_{v}+\beta \gamma+t L, \\
b_{u}+b^{2}+a \beta+s M & =\beta_{v}+b \alpha+\beta \delta+p+n L, \\
c_{u}+b c+a p+f M & =p_{v}+c \alpha+q \beta+g L, \\
M_{u}+a L+(b+A) M & =L_{v}+B L+\alpha M+\beta N, \\
t_{u}+t \alpha+a n+m B+g & =m_{v}+a m+s \gamma+t A, \\
g_{u}+p t+c n+f B & =f_{v}+q s+c m+g A, \\
B_{u}+t L+n M & =A_{v}+s N+m M
\end{aligned}
$$

and those obtainable therefrom by the substitution (3).

The lines $x y$ joining pairs of corresponding points $P_{x}, P_{y}$ of the surfaces $S_{x}, S_{y}$ form a congruence, the developables $\dagger$ of which are given by

$$
s d u^{2}-(m-n) d u d v-t d v^{2}=0 .
$$

The focal points of a line $x y$ are the points $\eta, \zeta$ given by

$$
\eta=y+k_{1} x, \quad \zeta=y+k_{2} x,
$$

* Lane, op. cit., p. 183 et seq.

† Ibid., p. 181. 
where $k_{1}, k_{2}$ are the roots of the equation

$$
k^{2}+(m+n) k+m n-s t=0 .
$$

It is known that the asymptotic curves on $S_{x}$ are parametric in case $L=N=0$. Let us suppose from now on that this condition is satisfied, and in order that the developables of the congruence of lines $x y$ be determinate and intersect $S_{x}$ in a conjugate net we shall suppose $m=n, s t \neq 0$.

It is not difficult to calculate the system of equations corresponding to (22) when the roles of $x$ and $y$ are interchanged. We shall compute all of the coefficients of such a system later, but at the moment the only coefficients that are needed are those corresponding to $L, M$, and $N$ which are indicated by dashes and given* by the following formulas:

$$
\begin{aligned}
\bar{L} & =f_{u}+n p+c s+\bar{A} c_{11}+\bar{B} c_{12}, \\
\bar{M} & =f_{v}+c n+q s+\bar{A} c_{31}+\bar{B} c_{32} \\
& =g_{u}+c n+p t+\bar{B} c_{41}+\bar{A} c_{42}, \\
\bar{N} & =g_{v}+n q+c t+\bar{B} c_{21}+\bar{A} c_{22},
\end{aligned}
$$

wherein the coefficients $c_{i j}$ are defined by placing

$$
\begin{array}{ll}
c_{11}=n_{u}+f+n \alpha+a s, & c_{12}=s_{u}+n \beta+b s, \\
c_{21}=n_{v}+g+n \delta+b t, & c_{22}=t_{v}+n \gamma+a t, \\
c_{31}=n_{v}+a n+s \gamma, & c_{32}=s_{v}+f+b n+s \delta, \\
c_{41}=n_{u}+b n+t \beta, & c_{42}=t_{u}+g+a n+t \alpha,
\end{array}
$$

and where

$$
\Delta \bar{A}=s g-n f, \quad \Delta \bar{B}=t f-n g, \quad \Delta=n^{2}-s t \neq 0 .
$$

The parametric curves on $S_{y}$ form a conjugate net $N_{y}$ in case $\bar{M}=0$. We shall suppose from now on that this condition is satisfied, and in order that $S_{\nu}$ may be non-developable we shall suppose $\bar{L} \bar{N} \neq 0$. The developables of the congruence of lines $x y$ intersect $S_{y}$ in a conjugate net in case

$$
t \bar{L}-s \bar{N}=0,
$$

a condition which we shall suppose from now on to be satisfied.

It is possible to simplify system (22) still more by a transformation of the form

$$
x=\lambda \bar{x}, \quad y=\mu \bar{y} .
$$

* Lane, op. cit., p. 185. 
The effect of this transformation on the coefficients $f, g, A, B$ is found to be given by the formulas

$$
\begin{aligned}
\mu \bar{f} & =\lambda\left(f+s \lambda_{u} / \lambda\right), & \mu \bar{g} & =\lambda\left(g+t \lambda_{v} / \lambda\right), \\
\bar{A} & =A-\mu_{u} / \mu, & \bar{B} & =B-\mu_{v} / \mu .
\end{aligned}
$$

The last of (23) shows that $\mu$ can be chosen so that $\bar{A}=\bar{B}=0$. We shall suppose from now on that this choice has been made. A condition necessary and sufficient that $\lambda$ can be chosen so that $\bar{f}=\bar{g}=0$ is

$$
(f / s)_{u}=(g / t)_{v} .
$$

By means of (23) and (26) this condition can be shown to be equivalent to (27). We shall suppose from now on that this choice of $\lambda$ has been made.

When $f=g=A=B=0$, the line $h$ of intersection of the tangent planes at two corresponding points $P_{x}, P_{y}$ of the surfaces $S_{x}, S_{y}$ joins the points $P_{\rho}, P_{\sigma}$ defined by

$$
\begin{aligned}
& \rho=y_{u}=x_{v}+n x_{u} / s \\
& \sigma=y_{v}=x_{u}+n x_{v} / t,
\end{aligned}
$$

as is seen on inspecting the last two of equations (22).

When $u, v$ vary, the line $h$ generates a congruence $\rho \sigma$, whose developables will now be determined. If, as the point $P_{x}$ describes a curve of the family $d v-\lambda d u=0$ on the surface $S_{x}$, the line $h$ generates a developable of the congruence $\rho \sigma$, and if the point $P_{\zeta}$ defined by

$$
\zeta=\rho+k \sigma
$$

( $k$ scalar)

is the corresponding focal point of the line $h$, then $h$ is tangent to the locus of the point $P_{\zeta}$; consequently the derivative $\zeta^{\prime}$ may be expressed as a linear combination of $\rho, \sigma$ only. But by actual calculation it is found that $\zeta^{\prime}$ appears as a linear combination of $x, \rho, \sigma, y$. Setting equal to zero the coefficients of $x, y$ therein, we obtain conditions on the functions $k, \lambda$ necessary and sufficient that the line $h$ may generate a developable of the congruence $\rho \sigma$ and have $P_{\zeta}$ for focal point, namely,

$$
\begin{aligned}
& (c s t+n p t)+(c n s+p s t) k+(q s t+n c t) \lambda+(c s t+n q s) k \lambda=0, \\
& s t+n s k+n t \lambda+s t k \lambda=0 .
\end{aligned}
$$

Elimination of $k$ and substitution of $d v / d u$ for $\lambda$ give the differential equation of the developables of the congruence $\rho \sigma$, namely,

$$
p d u^{2}-q d v^{2}=0 \text {. }
$$


A necessary and sufficient condition that the developables of the congruence $\rho \sigma$ be indeterminate is seen from (32) to be

$$
p=q=0 \text {. }
$$

We shall suppose from now on that this condition is satisfied. As a result of the conditions which we have thus far imposed we find from (26) that

$$
\bar{L}=c s, \quad \bar{M}=c n=0, \quad \bar{N}=c t .
$$

In view of the previous assumptions it is therefore evident from (34) that $m=n=0, c \neq 0$.

The most general transformation of the form (28) which leaves the form of system (22) invariant, has $\lambda$ and $\mu$ constant. The only coefficients not absolutely invariant under such a transformation are $s, t, M$, for which we find

$$
\bar{s}=\lambda s / \mu, \quad \bar{t}=\lambda t / \mu, \quad \bar{M}=\mu M / \lambda .
$$

It is not difficult to show by means of the integrability conditions (23) that

$$
M_{u} / M=c_{u} / c, \quad M_{v} / M=c_{v} / c,
$$

and from these results it is evident that

$$
M=k c
$$$$
\text { ( } k=\text { const.). }
$$

It is therefore possible by a suitable choice of $\lambda$ and $\mu$ to make the constant appearing in (36) equal to unity. We thus reach the following conclusion.

Any system (22) such that every pair of integral surfaces is projectively parallel in the modified sense can be reduced to the form

$$
\begin{array}{rlr}
x_{u u} & =\alpha x_{u}+\beta x_{v}, \\
x_{u v} & =M(x+y)+a x_{u}+b x_{v}, \\
x_{v v} & =\gamma x_{u}+\delta x_{v}, \\
y_{u} & =s x_{v}, \quad y_{v}=t x_{u}
\end{array}
$$

The parametric net $N_{x}$ is the asymptotic net and the parametric net $N_{y}$ is conjugate.

The integrability conditions for system (37) are found to be

$$
\begin{aligned}
a_{u}+a b+c & =\alpha_{v}+\beta \gamma, \\
b_{u}+b^{2}+a \beta+s M & =\beta_{v}+b \alpha+\beta \delta, \\
M_{u}+b M & =\alpha M, \\
t_{u}+t \alpha & =s \gamma,
\end{aligned}
$$

and the formulas obtainable from these by the substitution (3). 
The system of equations corresponding to (37) when the roles of $x$ and $y$ are interchanged is found to be

$$
\begin{aligned}
y_{u u} & =\bar{L}(x+y)+\bar{\alpha} y_{u}+\bar{\beta} y_{v}, \\
y_{u v} & =\bar{a} y_{u}+\bar{b} y_{v}, \\
y_{v v} & =\bar{N}(x+y)+\bar{\gamma} y_{u}+\bar{\delta} y_{v}, \\
x_{u} & =\bar{s} y_{v}, \quad x_{v}=\bar{t} y_{u},
\end{aligned}
$$

where

$$
\begin{aligned}
& \bar{L}=c s, \quad \bar{\alpha}=b+s_{u} / s, \quad \bar{\beta}=a s / t, \\
& \bar{a}=\beta t / s, \quad \bar{b}=\gamma s / t, \\
& \bar{N}=c t, \quad \bar{\gamma}=b t / s, \quad \bar{\delta}=a+t_{v} / t, \\
& \bar{s}=1 / t, \quad \bar{t}=1 / s .
\end{aligned}
$$

It is evident that the asymptotic curves which are parametric on $S_{x}$ correspond to the curves of the parametric conjugate net on $S_{y}$. The asymptotic curves on $S_{y}$ are given by

$$
\bar{L} d u^{2}+\bar{N} d v^{2}=0 .
$$

By use of (40) this equation becomes

$$
s d u^{2}+t d v^{2}=0
$$

which defines the associate conjugate net of the net in which the developables of the congruence of lines $x y$ intersect $S_{x}$. Thus since the asymptotic curves on each of the two surfaces $S_{x}, S_{\nu}$ correspond to a conjugate net on the other, we therefore reach the following conclusion:

Any system (22) such that every pair of integral surfaces is projectively associate in the modified sense, can be reduced to the form (37).

The last two equations of (37) show that the tangent to an asymptotic $u$-curve (v-curve) through $P_{x}$ on $S_{x}$ intersects the tangent to the $v$-curve ( $u$-curve) of the parametric conjugate net through $P_{y}$ on $S_{y}$ in a point which lies in a fixed plane. Since statements similar to the preceding can be made by choosing a conjugate net as parametric on $S_{x}$ and the asymptotic net as parametric on $S_{y}$, we therefore have a projective generalization of the theorem* of Eisenhart:

\footnotetext{
* Eisenhart, op. cit., p. 381.
} 
The tangents to the asymptotic curves on one of two projectively associate surfaces meet the tangents to the curves conjugate to the corresponding curves on the other surface in points of a fixed plane.

Inspection of (25) now shows that the two focal points $P_{\eta}, P_{\zeta}$ of a line $x y$ are given by

$$
\eta=y+(s t)^{1 / 2} x, \quad \zeta=y-(s t)^{1 / 2} x .
$$

The cross ratio of the points $P_{x}, P_{y}$ and the two focal points of the generator of the conjugate congruence is given by

$$
(x, y, \eta, \zeta)=\left(\infty, 0,(s t)^{1 / 2},-(s t)^{1 / 2}\right)=-1 .
$$

Corresponding points $P_{x}, P_{y}$ of two projectively associate surfaces $S_{x}, S_{y}$ are separated harmonically by the focal points of the line joining them.

If local coordinates $x_{1}, \cdots, x_{4}$ based on the tetrahedron $x, x_{u}, x_{v}, y$ with suitably chosen unit point are introduced, the first and second focal planes of a line $x y$ are found to have the local equations

$$
x_{2}-(t / s)^{1 / 2} x_{3}=0, \quad x_{2}+(t / s)^{1 / 2} x_{3}=0 .
$$

Therefore the planes $x_{2}=0, x_{3}=0$ containing $a$ line $x y$ and the asymptotic tangents through $P_{x}$ on $S_{x}$ separate the first and second focal planes of the line $x y$ harmonically.

The developables of the congruence of lines $x y$ intersect $S_{x}$ in a conjugate net whose differential equation may be written

$$
s d u^{2}-t d v^{2}=0 .
$$

Similarly, the developables intersect $S_{\nu}$ in the conjugate net given by

$$
\bar{s} d u^{2}-\bar{t} d v^{2}=0 .
$$

When reference is made to (40) it is evident that these curves on $S_{x}$ and $S_{y}$ correspond.

Southwestern College,

MeMpHIS, TENN. 